\title{
Direct Estimation of Cardiac Bi-ventricular Volumes with Regression Forests
}

\author{
Xiantong Zhen ${ }^{1}$, Zhijie Wang ${ }^{1}$, Ali Islam ${ }^{3}$, Mousumi Bhaduri ${ }^{4}$, Ian Chan $^{4}$ and \\ Shuo $\mathrm{Li}^{1,2}$ \\ 1 The University of Western Ontario, London, ON, Canada \\ ${ }^{2}$ GE Healthcare, London, ON, Canada \\ 3 St. Joseph's Health Care, London, ON, Canada \\ ${ }^{4}$ London Health Sciences Centre, ON, Canada
}

\begin{abstract}
Accurate estimation of ventricular volumes plays an essential role in clinical diagnosis of cardiac diseases. Existing methods either rely on segmentation or are restricted to direct estimation of the left ventricle. In this paper, we propose a novel method for direct and join$\mathrm{t}$ volume estimation of bi-ventricles, i.e., the left and right ventricles, without segmentation and user inputs. Based on the cardiac image representation by multiple and complementary features, we adopt regression forests to jointly estimate the two volumes. Our method is validated on a dataset of 56 subjects with a total of $3360 \mathrm{MR}$ images which shows that our method can achieve a high correlation coefficient of around 0.9 with manual segmentation obtained by human experts. With our proposed method, the most daily-used estimation of cardiac function, e.g., ejection fraction, can be conducted in a much more efficient, accurate and convenient way.
\end{abstract}

\section{Introduction}

Cardiac ventricular volumes provides an important measurement for assessment of cardiac functions and diagnosis of cardiac abnormalities. Conventional methods of estimating ventricular volumes depend on the results of manual or automatic segmentation. However, manual segmentation of MRI sequences is extremely time-consuming, subjective and highly non-reproducible. While automatic segmentation which has been extensively researched in the last a few decades is still regarded as a challenging and computationally expensive task, and remains unsolved, especially for the right ventricle (RV) [1], [2]. Direct estimation of cardiac ventricular volumes becomes increasingly attractive due to its efficiency and clinical significance. In recent work by Afshin et al. [3], left ventricle (LV) volumes are calculated by using image statistics, i.e., Bhattacharyya, similarity between image distributions, however intensive user inputs including two boxes, i.e., one inside LV cavity and one enclosing the cavity, are required. In addition, due to the strong assumption of correlation between the considered statistics and LV cavity areas the method is restricted to the LV, and therefore can not be generalised to the RV. In [4] [5], a Bayesian model is used for 
bi-ventricular volume estimation which however highly relies on a large set of segmented data.

Clinically, the RV has long been overlooked while its dysfunction also serves as an indicator of heart and lung disease and can adversely affect the function of the LV [6]. Technically, the RV has also been less researched compared to the LV because of the fundamental complex structural geometry of the RV, i.e., the more complex deformations of RV chamber in $3 \mathrm{D}$, crescent-shaped structure of large variation and the presence of papillary muscles [2]. However, simultaneous analysis of the LV and RV, e.g., bi-ventricular heart failure, can provide more meaningful and comprehensive assistance for diagnosis of cardiac diseases [7]. Therefore, direct and joint estimation of bi-ventricular volumes would be valuable for accurate, efficient and convenient assessment of cardiac functions, which has not been addressed yet due to the following challenges. Bi-ventricles exhibit great variability of cardiac images from gray levels to structure shapes as shown in Fig. 1. The myocardium and the papillary muscles inside the cavity (3 in the RV and 2 in the LV) are connected and have almost the same intensity [8]. The shape of ventricles varies across patients, over time and along the long axis which makes it extremely difficult for accurate analysis of bi-ventricular volumes. Even more challenges arise from the complex functional and geometrical interference and interdependency between the right and left ventricle.

In this work, we propose a novel method for direct and joint bi-ventricular volume estimation. Our method consists of two main steps: cardiac image representation and random forest regression. Specifically, we propose a cardiac image representation by multiple features including pyramidal Gabor features (PGF), histogram of oriented gradients (HOG) [9] and appreance. Random forests [10], [11] are learned from training samples with ground truth obtained by human experts and then employed for joint estimation of bi-ventricular volumes in unseen images. Our work contributes in three folds: (1) the first direct and joint estimation of cardiac bi-ventricular volumes without segmentation; (2) customised and adapted multiple features for cardiac image representation; (3) the use of regression forests for efficient bi-ventricular volume estimation.
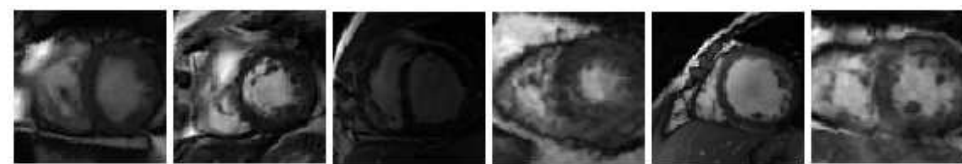

Fig. 1. The illustration of MR images with bi-ventricles.

\section{Direct Bi-ventricular Volume Estimation}

To overcome the challenges mentioned above, an effective representation of cardiac images is desired for direct and joint estimation. We propose multiple features for cardiac image representation which benefits in the following aspects: it 
1) provides rich and complementary information closely related to bi-ventricular volumes; 2) can capture substantial features to handle the combinatorial variability of bi-ventricles; 3) is more flexible and generalisable than segmentation-based methods.

The multiple heterogeneous features generate a high-dimensional representation of cardiac images which demands the regressor to be efficient for estimation. Regression forests have shown the efficiency and effectiveness of modelling complex relationships between high-dimensional input features and continuous outputs [10] and are particularly suitable for bi-ventricular volume estimation because they 1) provide a natural and efficient way for regression based on multiple features because of their ability of handling heterogeneous features [12] and fast implementation; 2) produce highly accurate predictions and can avoid overfitting with a very large number of input variables [13]; 3) are optimised to choose the most relevant features to bi-ventricular volumes specifically due to the use of feature selection.

\subsection{Cardiac image representation}

An effective representation of cardiac images should be able to capture features that are closely related to bi-ventricular volumes. Bi-ventricular volumes as shown in Fig. 2 (a) are determined by their cavity shapes and intensity. We use PGF, HOG and appearance because of their effectiveness and mutual complementarity: PGF can effectively capture edges and boundaries in multiple scales and orientations; HOG describes the main shape of bi-ventricular cavities; and the appearance, i.e., pixel intensity, carries the fundamental layout of bi-ventricles. Combination of them provides a comprehensive and informative representation of cardiac images.

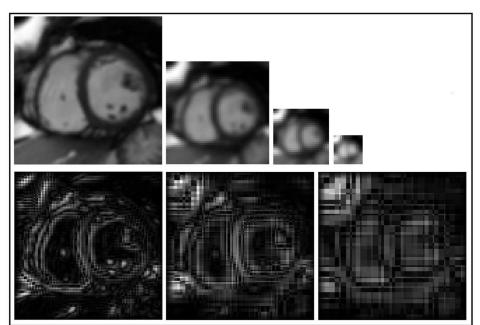

(a)

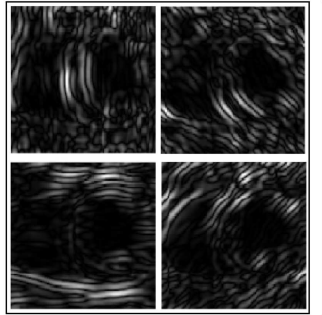

(b)

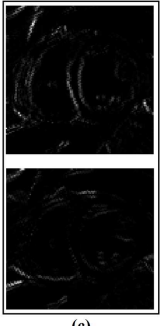

(c)

Fig. 2. (a) Gaussian pyramid (upper) and Laplacian pyramid (bottom). (b) Gabor filtered in 4 orientations. (c) squared gradients along $\mathrm{X}$ (upper) and $\mathrm{Y}$ (bottom) axis, respectively.

Pyramidal Gabor features (PGF) are specially developed via multiscale and multi-orientation analysis to capture sufficient features related to bi- 
ventricular shapes which determine cavity areas. The multi-scale analysis is realised by the Laplacian pyramid and Gabor filters are adopted to incorporate multi-orientation information, the key cue of shapes. The Laplacian pyramid is constructed by differencing adjacent levels of a Gaussian pyramid as shown in the upper row of Fig. 2 (a). At each level of the Laplacian pyramid, edges with different scales are intensified as shown in the bottom of Fig. 2 (a). On top of each level of the Laplacian pyramid, we then design a bank of Gabor filters to detect edges of bi-ventricles in multiple orientations. Fig. 2 (b) illustrate the outputs of Gabor filtering in four orientations. Compared with gradients in Fig. 2 (c), much more details related to edges of ventricles are intensified. The 2D Gabor mother function is defined as:

$$
F(x, y)=e^{-\frac{x_{0}^{2}+y_{0}^{2}}{2 \sigma^{2}}} \cos \frac{2 \pi x_{0}}{\lambda}
$$

where $x_{0}=x \cos \theta+y \sin \theta, y_{0}=-x \sin \theta+y \cos \theta$, and the range of $\sigma$ decides the scales of Gabor filters and $\theta$ determines orientations.

Histogram of oriented gradients (HOG) [9] describes the main shapes of bi-ventricles which upholds invariance to geometric and photometric transformations by operating on localized cells. Fig. 2 (c) shows the squared gradients along vertical and horizontal directions which are used for the computation of the HOG descriptor. The rough outlines of bi-ventricular cavities are successfully captured.

Appearance, i.e., the intensity of pixels, carries fundamental information of ventricular cavities as the intensity within cavities is brighter than myocardium and most of the background. In addition, flattening the pixels as feature vector can also provide us the layout information of bi-ventricles.

\section{$2.2 \quad$ Regression Forests}

Random forests [10] are an ensemble of decision trees which combine the ideas of bagging and the random feature selection, and recently started to attract interest in medical image analysis [14]. They can effectively deal with the highdimensional representation due to the feature selection and possess innate ability to handle heterogeneous features from different sources, offering a prime option for bi-ventricular volume estimation with multiple features. Coupled with the cardiac image representation by multiple features, random forests build a strong tool for bi-ventricular volume estimation.

Given a multivariate input $\mathbf{v}$ which is the feature vector extracted from each MR image, regression forests are learned to associate $\mathbf{v}$ with a continuous multivairate label $\mathbf{y}$ which is the cavity areas and then employed to predict cavity areas in unseen MR images.

Training We build decision trees using the adapted algorithm from [10]. Each internal node of a tree is associated with a split function. The training process is to construct each tree with a randomly selected training subset. Note that only a subset of features are used for each decision tree which are fixed for 
prediction. An example of trained random forests are shown in Fig. 3. The split function at a split node $j$ is formulated as a function with binary outputs

$$
h\left(\mathbf{v}, \boldsymbol{\theta}_{\boldsymbol{j}}\right): R^{d} \times \mathcal{T} \rightarrow\{0,1\}
$$

where $\mathbf{v}$ is the input feature vector, $\mathcal{T}$ represents the space of all split parameters [11] and $\boldsymbol{\theta}_{\boldsymbol{j}}$ is the function parameter associated with the $\boldsymbol{j}$-th node and can be trained by minimizing a least-squares error function $I[10]$ at the $j$-th split node:

$$
\boldsymbol{\theta}_{\boldsymbol{j}}=\arg \max _{\boldsymbol{\theta} \in \mathcal{T}} I\left(S_{j}, \boldsymbol{\theta}\right)
$$

where $S_{j}$ is a subset of training samples associated with the $j$-th node. The data point $\mathbf{v}$ arriving at the split node is sent to its left or right child node according to the result of the split function.

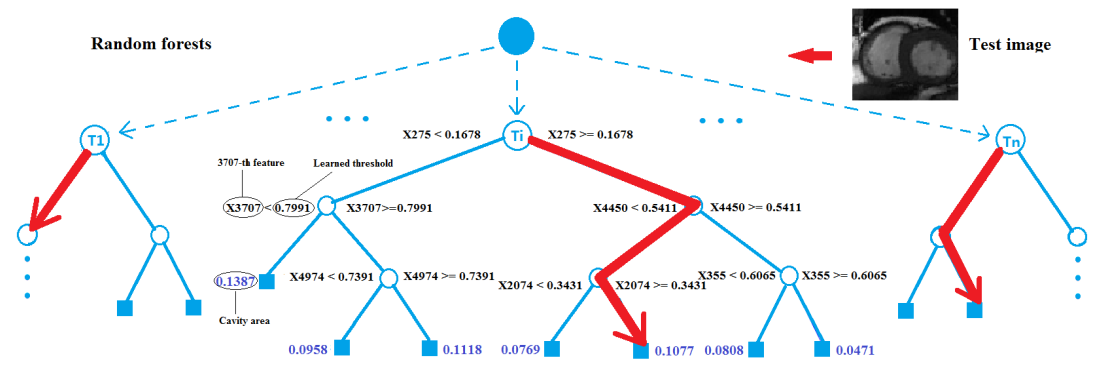

Fig. 3. Illustrated are the random forests comprised of $n$ decision trees $\left\{T_{1}, \ldots, T_{i}, \ldots, T_{n}\right\}$ learned from the training set. Test images can be quickly predicted by several simple comparison operations. $X \#$ indicates the \#-th feature.

Prediction Given a previously unseen MR image v, our objective is to predict bi-ventricular volumes using the learned random forests. As shown in Fig. 3, we pass $\mathbf{v}$ through each tree starting from the root of each decision tree $T_{i}$, send to the left/right child by applying the split function, and stop when $\mathbf{v}$ reaches a leaf node of the tree. The simple comparison operation on each split node makes the prediction extremely fast and efficient. Given the $t$-th tree in a forest, the associated leaf output takes the form of a density probability function $p_{t}(\mathbf{y} \mid \mathbf{v})$. The forest output is the average of all tree outputs

$$
p(\mathbf{y} \mid \mathbf{v})=\frac{1}{T} \sum_{t=1}^{T} p_{t}(\mathbf{y} \mid \mathbf{v})
$$

where $T$ is the number of trees in the forest. 


\section{Experiments and results}

We conduct experiments on clinical MR images and the estimated volumes are quantitatively evaluated by correlating with those obtained from manual segmentation by an expert. In our experiments, a set of (3360 in total) 2D short-axis cine MR images from 56 clinical subjects including both normal and abnormal cases were used. These images were acquired on a $1.5 \mathrm{~T}$ scanner with fast imaging employing steady-state acquisition (FIESTA) image sequence mode, using these acquisition parameters: $\mathrm{TR}=2.98 \mathrm{~ms}, \mathrm{TE}=1.2 \mathrm{~ms}$, flip angle $=30$ degrees, and slice thickness $=10 \mathrm{~mm}$. Each subject contains 20 frames throughout a cardiac cycle. In each frame, three representative slices, i.e., apical, mid-cavity and basal, are selected following the standard AHA prescriptions [15] for validation, and their manual segmentations are used as the benchmark. We estimate cavity areas of the LV and RV in MR images, and the volumes are computed by integrating LV/RV cavity areas in the sagittal direction. A single region of interest (ROI) rather than two individual ones is placed to enclose the LV and RV in an MR image, which is obtained by the template matching and hough transform [5]. We employ a leave-one subject-out validation approach.

\section{$3.1 \quad$ Results}

The comparison of estimated volumes with the manual segmentation is depicted in Fig. 4 (a) and (e) which are the correlations between estimated volumes and manually obtained volumes for the LV and RV, respectively. Despite of the challenges in joint estimation of bi-ventricular volumes, the proposed method achieves a correlation coefficient of $\mathbf{0 . 9 2 2}$ for the LV, and can yield $\mathbf{0 . 8 7 4}$ for the RV which has much greater geometrical complexity than the LV. The estimation errors are $0.0129 \pm 0.0115$ (LV) and $0.0155 \pm 0.0127(\mathrm{RV})$. The result$\mathrm{s}$ confirm multiple feature representation overcomes the great variability

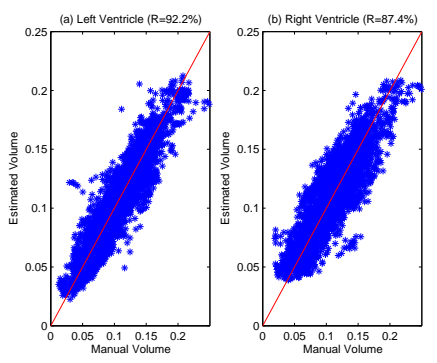

Fig. 4. Illustration of correlation between estimated and manually obtained biventricular volumes. $R$ is the correlation coefficient.

and geometrical complexity of bi-ventricles, and validate the effectiveness of the proposed method for direct and joint estimation of the two volumes.

The complementarity of PGF, HOG and appearance is demonstrated in Table 1. The results show that the combined feature comprehensively represent cardiac images for the benefit of estimating bi-ventricular volumes. The effectiveness of the proposed method are further demonstrated by the results on cardiac apical, mid-cavity and basal which are plotted in Fig. 4 (b)-(d) and (f)-(h) for the LV and RV, respectively. The proposed method can achieve a correlation coefficient 
of more than $\mathbf{0 . 9}$ for LV apical, although it is much more challenging than midcavity and basal due to the disturb of papillary muscles.

To show the potential of the proposed method for quantification of cardiac functions, we plot the volumes of the 20 frames from one subject in Fig. 5 (a) and (b) for left and right ventricles, respectively. The proposed method successfully captures the dynamic pattern of volumes in a cardiac cycle which will be extremely useful for clinical assessment of cardiac functions, e.g., ejection fraction (EF). Moreover, this can now be done directly without segmentation and user inputs.

Table 1. The correlation coefficients between manually obtained and estimated volumes with different features.

\begin{tabular}{l|llll}
\hline Feature & Appearance & HOG & PGF & Combined \\
\hline Left ventricle & 0.793 & 0.811 & 0.845 & $\mathbf{0 . 9 2 2}$ \\
Right ventricle & 0.780 & 0.804 & 0.836 & $\mathbf{0 . 8 7 4}$ \\
\hline
\end{tabular}

Fig. 6 (a) and (b) depict the cases which the proposed method gives best estimation results with lowest errors for the LV and RV, respectively. Although the intensity and shapes of bi-ventricles varies greatly across different images, the proposed method has produced nearly perfect estimation with errors close to zeros, which further shows the strength of the integration of multiple feature representation and random forests for bi-ventricular volume estima-
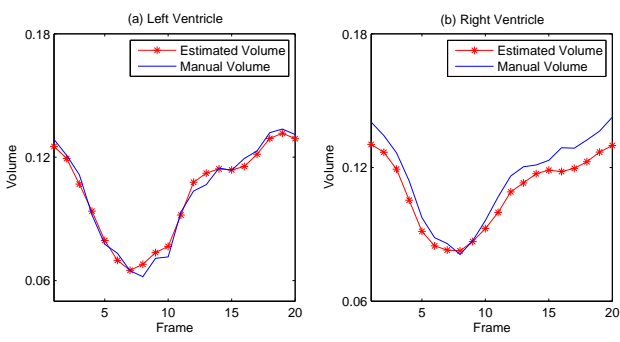

Fig. 5. Illustration of dynamics of bi-ventricular volumes in one cardiac cycle. (a) left ventricle; (b) right ventricle. tion.

\section{Conclusion}

In this paper, we proposed a novel method for directly estimating volumes of both left and right ventricles jointly. We remove the need of segmentation and formulate volume estimation as a regression problem. Cardiac bi-ventricles are represented by multiple and complementary features and regression forests are employed for volume estimation. Experimental results show that the proposed method can predict bi-ventricular volumes with high correlations with those 


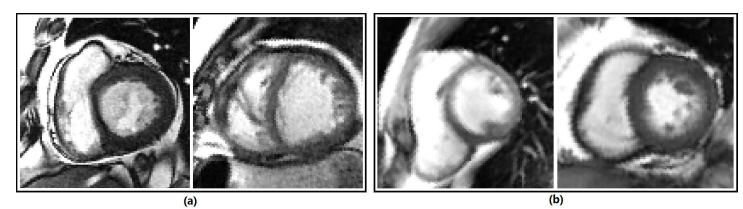

Fig. 6. The best estimations for the LV (a) and RV (b).

obtained manually by an expert, which validates the effectiveness of the proposed method for direct and joint estimation of bi-ventricular volumes. Our method enables a more efficient and accurate way for assessment of cardiac functions.

\section{References}

1. C. Petitjean, J.-N. Dacher, A review of segmentation methods in short axis cardiac MR images, Medical Image Analysis 15 (2) (2011) 169-184.

2. C. M. Nambakhsh, T. M. Peters, A. Islam, I. B. Ayed, Right ventricle segmentation with probability product kernel constraints, in: MICCAI, 2013, pp. 509-517.

3. M. Afshin, I. B. Ayed, A. Islam, A. Goela, T. M. Peters, S. Li, Global assessment of cardiac function using image statistics in MRI, in: MICCAI, 2012, pp. 535-543.

4. Z. Wang, M. Salah, I. Ayed, A. Islam, A. Goela, S. Li, Bi-ventricular volume estimation for cardiac functional assessment, in: RSNA, 2013.

5. Z. Wang, M. Ben Salah, B. Gu, A. Islam, A. Goela, S. Li, Direct estimation of cardiac bi-ventricular volumes with an adapted bayesian formulation, IEEE TBME (2014) 1251-1260.

6. I. Haber, D. N. Metaxas, L. Axel, Three-dimensional motion reconstruction and analysis of the right ventricle using tagged MRI, Medical Image Analysis 4 (4) (2000) 335-355.

7. X. Lu, Y. Wang, B. Georgescu, A. Littman, D. Comaniciu, Automatic delineation of left and right ventricles in cardiac MRI sequences using a joint ventricular model, in: FIMH, 2011, pp. 250-258.

8. I. Ben Ayed, S. Li, I. Ross, Embedding overlap priors in variational left ventricle tracking, IEEE TMI 28 (12) (2009) 1902-1913.

9. N. Dalal, B. Triggs, Histograms of oriented gradients for human detection, in: CVPR, Vol. 1, 2005, pp. 886-893.

10. L. Breiman, Random forests, Machine learning 45 (1) (2001) 5-32.

11. A. Criminisi, J. Shotton, Decision Forests for Computer Vision and Medical Image Analysis, Springer Publishing Company, Incorporated, 2013.

12. R. Johnson, T. Zhang, Learning nonlinear functions using regularized greedy forest, IEEE TPAMI 36 (2014) 942-954.

13. G. Biau, Analysis of a random forests model, JMLR 98888 (2012) 1063-1095.

14. A. Criminisi, J. Shotton, D. Robertson, E. Konukoglu, Regression forests for efficient anatomy detection and localization in CT studies, in: MICCAI-MCV, 2011, pp. 106-117.

15. M. D. Cerqueira, et al., Standardized myocardial segmentation and nomenclature for tomographic imaging of the heart a statement for healthcare professionals from the cardiac imaging committee of the council on clinical cardiology of the american heart association, Circulation 105 (4) (2002) 539-542. 